\title{
Design and Implementation of High-rise Buildings Based on GPS Po- sitioning Technology
}

\author{
Henan four construction co., Ltd. Kaifeng 475000, Henan
}

\section{Introduction}

GPS positioning technology was originally designed to provide real-time, accurate navigation services to land, sea and air, and can also be used for emergency communications, intelligence gathering and other military activities. However, with the continuous improvement of the technology, in addition to the above functions can be achieved, but also high-precision relative static positioning. Thus, GPS positioning technology has good development prospects.

So far, GPS positioning technology in the transportation, aerospace and other industries have been widely used, but also show a certain applicability, but its high-rise building construction has no systematic theoretical research and successful application examples. This paper studies the design and implementation of high-rise building based on GPS positioning technology, hoping to promote the effective combination of GPS positioning technology and construction industry, strengthen the construction effect and improve the construction efficiency. As the construction industry has no standard definition for high-rise or super-high-rise buildings, this article is referred to as high-rise buildings in subsequent analysis.

\section{Design Technology of GPS Positioning High-rise Building}

\subsection{Design basis}

GPS network technology design is generally based on measurement procedures and measurement tasks.
(1) GPS measurement procedures

GPS measurement procedures is the mapping of various departments to develop technical specifications.

\section{(2) Measuring the task book}

The measurement task book, which is the technical contract required by the customer or the construction management, is instructive, and the requirements on the measurement range and measurement accuracy are put forward, which limits the time and effect of the project completion time.

Under normal circumstances, in the design of GPS program, according to the measurement contract provides GPS network accuracy and the index value, combined with measurement procedures and field survey to confirm the connection between each other, measuring frequency and measurement time.

\subsection{Positioning accuracy design}

The accuracy of all GPS network positioning is affected by the use of the net. Accuracy is a measure of GPS network technology design of the main indicators, is divided into five levels, the GPS network pattern, measurement methods and data processing has a direct role. In the construction, to combine the specific needs of the owners and the allocation of resources to design to confirm the accuracy, but also learn from the previous building experience and building standards. $(\mathrm{Mm})$ of the pitch of the GPS baseline vector; a rep

\begin{abstract}
: on the high-rise building construction, the benchmark observation, elevation control is an important content of quality control. Based on the goal of construction file quality and ensuring completion time, to enhance the accuracy and efficiency of observing and positioning in and to explore the suitable for high-rise building construction of scientific orientation observation. This thesis to research on based on GPS positioning technology of the high-rise building construction and implementation.
\end{abstract}

Key words: High-rise building construction; GPS positioning technology; design; implementation

Published online: 15th July, 2017

resents the fixed error $(\mathrm{mm})$ in the nominal accuracy of the GPS receiver; $b$ represents the GPS receiver $(\mathrm{Ppm} \cdot \mathrm{D})$; $\mathrm{d}$ represents the distance $(\mathrm{km})$ between two adjacent points of the GPS network).

\subsection{Graphic design}

Although the GPS network design by the owners of the most significant impact, but still by the cost, personnel, equipment and facilities, follow-up maintenance and other factors. Because of high-rise building complex and cumbersome, so to strengthen 
the emphasis on graphics design to meet the needs of owners and reduce resource consumption.

(1) The basic principles

(1) Under normal circumstances, GPS network is formed by the independent observation of the closed loop or composite lines, to enhance the level of the audit to enhance the stability of the network.

(2) The points within the GPS network and the ground control points overlap.

(3) GPS network points to overlap with the level of the point, do not overlap the point is usually combined with the specific circumstances through the level of measurement means to measure, or set the level of measurement points in the network to provide the basis for the ground level analysis.

(4) Based on the traditional way to measure the target, in the network around the points to set a good line of sight, to build the joint measurement direction.

\section{(2) Graphics type}

In general, GPS network non-synchronous observation edge to form a geometric pattern, including the triangle, star and ring (see Figure 1).

\subsection{Benchmark design}

It is a kind of WGS-84 coordinate system through GPS positioning system. However, high-rise building construction adopts the national coordinate system. Therefore, it is necessary to confirm the GPS system based on the coordinate system standard and initial Value, that is, GPS network based on the benchmark. Baselines are usually divided into position, orientation, and scale. The azimuth reference is usually confirmed by the initial azimuth angle and can also be confirmed by the baseline vector. The scale reference is usually confirmed by the ground electromagnetic wave, or by two or more initial points, and can also be confirmed by the baseline vector. The position reference is usually confirmed by the initial coordinate. It can be seen that the benchmark design of the GPS network is to confirm the location of the network benchmark.

\subsection{Line design}

Because the high-rise building GPS datum point is generally arranged in special position, such as building a center of gravity, structural axis, etc., the coordinate value of the building outside the temporary base point to receive GPS signal acquisition, the error is small, high precision. Based on the above work, you can work through the traditional measurement method.

\section{Preparation of foreign trade and technical design book}

2.1 High-rise building around the situation and data collection

(1) High-rise building around the situation to master

Grasp the situation around the highrise buildings generally indicate the survey and measurement area, that is, after receiving the task instructions or signed GPS positioning measurement task book, in accordance with the construction design program survey construction area, for the development of technical programs, cost estimates to provide the basis.

\section{(2) Data collection}

In accordance with the situation around the high-rise building to grasp the extent of the following information:

(1) Various drawings: construction drawings, topographic maps, ground level ups and downs, road facilities, etc.;

(2) Construction of regional traffic conditions, climatic conditions, topography and geology;

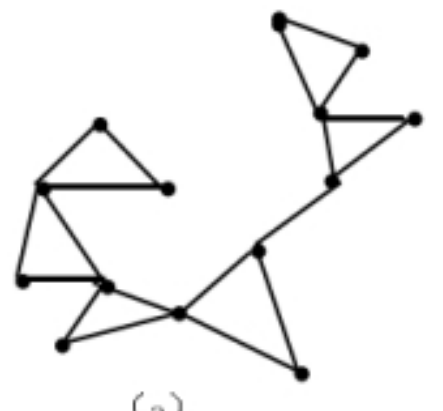

(a)

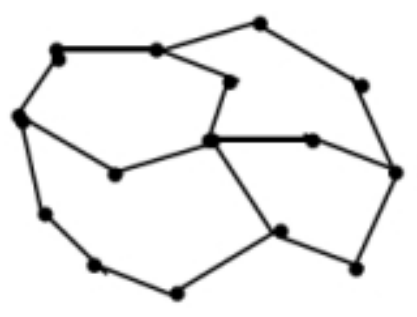

(b)

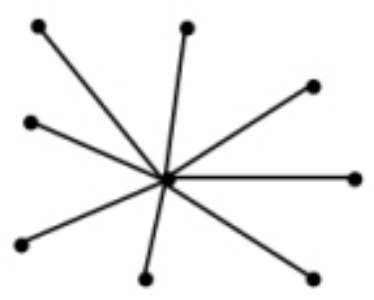

(c)

Figure 1 GPS network graphics type

a-Triangular mesh; b-Ring network; c-Star network 
(3) Various control points: level, GPS receiver, wire points, coordinates, etc.;

(4) Construction enterprise qualification: construction technology level, management level, construction personnel quality and so on.

2.2GPS positioning observation facilities and personnel preparation

(1) Set up the installation of related facilities, equipment, computers and so on;

(2) Installation of communication facilities and motorized facilities;

(3) Set up the installation of construction equipment, the development of the use of building materials budget;

(4) Organization of GPS positioning observation team, clear the duties of each staff;

(5) System positioning measurement budget.

2.3 GPS positioning observation plan development

GPS positioning of the field work is positioning observation. Before the development of observation, the development of field observation program is conducive to the collection of data, improve the positioning accuracy.

(1) To determine the basis for observation plan

(2) To develop observation plans

2.4 To develop technical design book

After the completion of the above work, to develop GPS positioning technology design book. In general, GPS positioning technology design books include:

(1) Construction requirements and work intensity

(2) The basic situation of high-rise buildings

(3) The layout of the program

(4) Election point observation
(5) Data analysis

(6) Other

\section{High-rise building construction GPS positioning of the imple- mentation of the field}

\subsection{Select points}

GPS positioning observation does not require visibility, and the network graphics structure flexibility is strong, so the relative positioning of the traditional way of positioning work, GPS positioning point selection is more convenient and simple. The completion of the selection work has a direct impact on the construction and observation accuracy of the construction works. Therefore, before starting the selection, we should not only grasp the surroundings of the building and the basic measurement and control structure, but also follow the following principles:

(1) To prevent the interference of electromagnetic fields;

(2) Points to be set in the field of vision is open, easy to install the location;

(3) Point layout near the terrain flat, can be stored for a long time.

\subsection{Embedding signs}

GPS network points are mostly set in the center of the standard stone position, to accurately lay the points. And the mark of the points to be stable, to ensure that the high-rise building in the cycle can be used effectively. Especially at the point outside the construction site area, to ensure the stability of the construction phase. The name of each point and the construction management to confirm the consultation, due to the construction phase of more personnel, and the construction process is generally responsible for the construction team, which virtually enhance the protection of the difficulty, in order to protect the point of stone, to try to avoid the construction area, while assigning someone responsible. After the points are buried, the following materials should be submitted:
(1) The name of each point;

(2) GPS network selection point map;

(3) Land acquisition approval documents and measurement power of attorney;

(4) Selection and placement of signs work summary.

\subsection{Observation work}

\section{(1) Basic technical requirements}

Compared with the traditional measurement methods, GPS positioning observation technology in the operating mode, measurement and other aspects of the requirements of more stringent, especially high-rise building construction.

\section{(2) Set up the antenna}

(1) In general, the antenna is placed on a tripod and coincides with the center of the sign, and there is no air bubbles in the base circle.

(2) Special circumstances, the antenna to be installed in the tripod back to the stage, to remove the top, to avoid blocking the GPS signal, this time to position signs projected on the back to the stage. If you cannot remove the top, it will lead to signal interruption, reduce GPS positioning accuracy, this situation applies to eccentric observation.

(3) Antenna direction should point to the north, but also consider the impact of electromagnetic fields, to reduce the phase center error.

(4) For high-rise building construction antenna set up to the antenna three fixed, to prevent fell to the ground damage.

\section{(3) Observation}

After the signal receiver collects the input data, the observer can understand and inquire the information according to the equipment operation manual, and cannot inquire any information without knowing the operation flow. In general, no parameters can be modified when the signal is received.

\section{(4) Observation records}


On the high-rise building construction GPS positioning observation, can take observation records and observation hand to record, any observation records should be strictly kept.

\subsection{Operating mode}

\section{(1) Static positioning mode}

Static positioning is usually used in the construction of high-rise building monitoring system, benchmarking and building positioning. This mode requires observation of the baseline to form a closed pattern structure (see Figure 2 below), thereby improving the positioning accuracy. In addition, fast and static positioning can be used to carry out high-rise building construction positioning (see Figure 3), this model can cut off the power to reduce energy consumption.
Dynamic positioning mode is generally divided into quasi-dynamic and dynamic positioning mode. The quasi-dynamic positioning mode is to select a reference point to install the receiver tracking satellite, install the mobile receiver at position 1 (see Figure 4 below), to ensure continuous tracking of the satellite, move the mobile receiver to positions 2,4 , ... to observe. The dynamic positioning mode is to continuously mount the receiver at a reference point to continuously track the satellite (see Figure 5 below) and then move the observations continuously. Both models are suitable for high-rise building construction.

\section{Conclusion}

GPS positioning technology as a new technology, has been in the engineering control measurement has been widely used, the technology has the unique advantages and advanced nature has been recognized by the majority of engineering staff. At this stage, China is in the great period of economic development, the extensive use of various types of engineering equipment and facilities, the application of new technology and new materials, but also to China's construction industry to show a thriving new scene. With the further research and popularization of GPS positioning technology, especially in the construction of high - rise buildings, GPS technology has been accumulated and improved in theoretical research, implementation methods, signal acceptance methods, signal processing and software development. Future GPS positioning technology will become a highrise building construction measurement positioning, deviation control

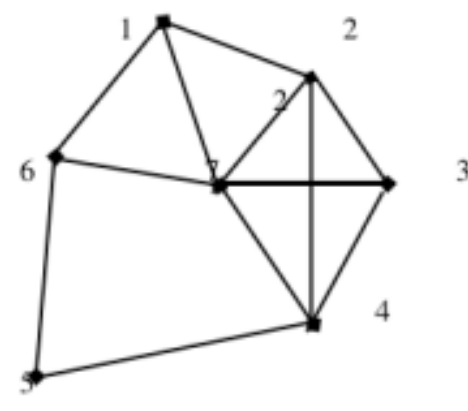

Figure 2 Static positioning

(2) Dynamic positioning mode

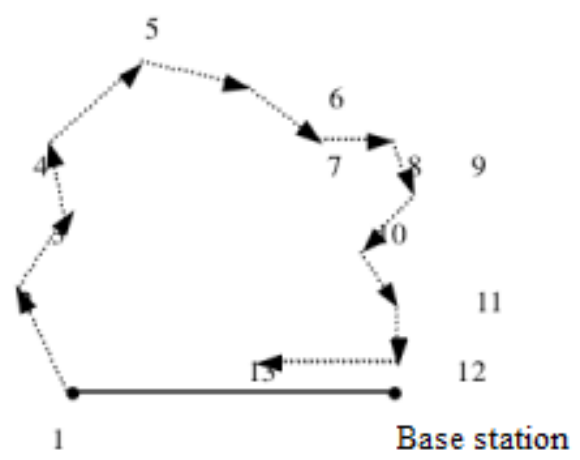

Figure 4 Quasi-dynamic positioning

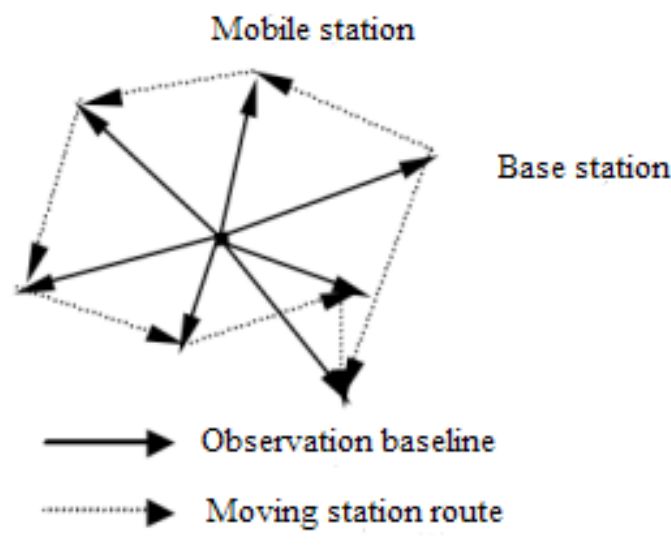

Figure 3 Fast static positioning

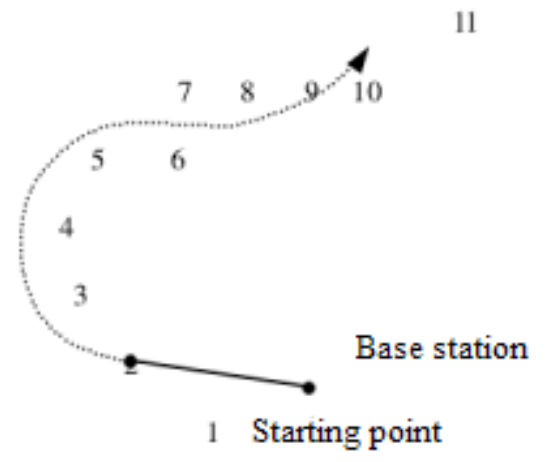

Figure 5 Dynamic positioning 
and other aspects of the widely used method.

\section{References}

[1] Guo Yingqi, Shi Daqi, Huang Shengxiang, Zhang Weicheng. Application of Wavelet Analysis in High Precision GPS Survey [J]. Surveying and Mapping Engineering .2009 (03)

[2] Wang Zhengxu, Du Zhixing, Dan Rui. Application of GPS Technology in Deformation Monitoring of High-rise Buildings [J]. Communication Survey .2009 (03)

[3] HUANG Sheng-xiang, LI Zhicheng. Multi-Equidistant Gray Modeling of Settlement Prediction of Engineering Buildings [J]. Acta Geographic Information. 2004 (01)

[4] Huang Sheng, Liu Jingnan, Liu Jilin. Application of Wavelet Analysis in Dynamic Monitoring of High-rise Buildings [J]. Journal of Surveying and Mapping .2003 (02)

[5] HUANG Sheng-xiang, LIU Jing- nan. Effect of Eliminating Noise in GPS Deformation Monitoring System [J]. Journal of Surveying and Mapping. 2002 (02)

[6] ZHANG Jin-cun, HUANG Shenghe. Application of Global Satellite Positioning Technology in Construction Engineering [J]. Construction Technology .2001 (02)

[7] Shen Xiaoping, Cao Haorong, Tang Qianlong. GPS Positioning Technology in the Application of Deformation Monitoring in the Building [J]. Geotechnical Foundation .2012 (02)

[8] Zhang Wenbin, Liu Fang. GPS Positioning Technology in the Use of Deformation Monitoring [J]. Jiangxi Building Materials .2015 (22)

[9] Guo Wen, Ding Gaoyuan. GPS Positioning Technology in the Shenzhen Metro Line 1 Continued Construction of the Completion of the Application of Surveying and Mapping [J]. Modern property (late) 2013 (12)

[10] Mi Hada Tie Cheeken. GPS Po- sitioning Technology in the Application of Highway Engineering Practice [J]. Silicon Valley .2013 (12)

[11] Ye Hu. GPS Positioning Technology in the Mining Area of the Application of Observation [J]. Science and Technology Communication .2013 (18)

[12] Wang Yan. Improve the GPS Positioning Technology Elevation Measurement Accuracy of the Elements and Methods [J]. Beijing Surveying and Mapping .2012 (02)

[13] Wu J T. Processing Mixed Pseudo-Range and Carrier Phase GPS Data. Manuscripta Geodaetica.1995

[14] Guo J J, Ge S. Reasearch of Displacement and Frequency of Tall Building under Wind Load Using GPS.ION GPS-1997.1997

[15] Ashkenazi V, Dodson A H, Moore $\mathrm{T}$, et al. Monitoring the Movements of Bridges by GPS. Proceedings of ION GPS-97.1997 